\title{
Detection of quantitative trait loci affecting the milk fatty acid profile on sheep chromosome 22: Role of the stearoyl-CoA desaturase gene in Spanish Churra sheep
}

\author{
M. García-Fernández, B. Gutiérrez-Gil, E. García-Gámez, J. P. Sánchez, and J. J. Arranz ${ }^{1}$ \\ Departamento de Producción Animal, Facultad de Veterinaria, Universidad de León, 24071, León, Spain
}

\begin{abstract}
Sheep milk fat contains several components that may provide human health benefits, such as monounsaturated fatty acids and conjugated linoleic acid (CLA). Most of the CLA in ruminant milk is synthesized in the mammary gland by the action of the enzyme stearoyl-CoA desaturase $(S C D)$ on circulating vaccenic acid (trans-11 C18:2; VA). Previous studies have found significant associations between polymorphisms in the $S C D$ gene and the fatty acid composition of ruminant products, including sheep milk. Based on this, we performed a quantitative trait loci (QTL) analysis of an ovine chromosome (22) that harbors the $S C D$ gene for effects on milk fatty acid composition traits and classical milk production traits. We identified a suggestive QTL influencing the CLA/VA ratio with the maximum statistic at position $26 \mathrm{cM}$ of the studied chromosome, whereas the $S C D$ gene has been mapped to position 41.6 $\mathrm{cM}$. The individual introduction of $4 S C D$ single nucleotide polymorphisms in the QTL model did not cause a reduction of the variance explained by the QTL, which suggests that the $S C D$ gene is not directly responsible for the detected effect in the Churra population studied herein. This conclusion was supported by the lack of any significant association identified between the 4 $S C D$ single nucleotide polymorphisms and the CLA/ VA ratio. This association analysis suggested a possible effect of the $S C D$ gene on milk fat percentage in Churra sheep. An independent confirmation of these primary results will be required before attempting its practical implementation in selection programs.
\end{abstract}

Key words: quantitative trait locus, milk fatty acids, stearoyl-CoA desaturase, dairy sheep

\section{INTRODUCTION}

The fatty acid (FA) composition of ruminant milk is an important variable that affects the nutritional value

Received June 16, 2009.

Accepted September 21, 2009.

${ }^{1}$ Corresponding author: jjarrs@unileon.es of dairy products and influences their manufacturing properties (Jensen et al., 1990; Bauman et al., 2006). Sheep milk is mainly consumed as cheese, which may be considered an unhealthy product by some consumers because of its high fat content. On average, sheep milk fat comprises more than 60\% saturated FA (SFA), $28 \%$ monounsaturated FA (MUFA), and 6\% polyunsaturated FA (PUFA; Cabiddu et al., 2003, 2005). However, it also contains several components that may provide benefits to human health, such as MUFA and conjugated linoleic acid (CLA). Conjugated linoleic acid is a collective term for isomers of linoleic acid with a conjugated double bond in several positions. The predominant CLA found in milk, cis-9, trans-11 CLA, has been shown to have several beneficial effects on human health because of its anticarcinogenic, antilipogenic, and immunomodulating activities (Pariza et al., 2000). More than $70 \%$ of the cis-9, trans-11 CLA in ruminant milk is synthesized in the mammary gland by the action of the enzyme stearoyl-CoA desaturase (SCD) on circulating vaccenic acid (trans-11 C18:2; VA) (Bauman et al., 2006). Stearoyl-CoA desaturase introduces a cis double bond between carbons 9 and 10 in a spectrum of different FA, which influences the amount of several unsaturated FA (Ntambi, 1995; Bauman et al., 1999). On the basis of this enzyme's biological function, the $S C D$ gene appears to be an interesting functional candidate to explain a portion of the genetic variability of milk FA composition in ruminant products. Several groups have reported significant associations between polymorphisms in this gene in cattle and the FA composition of carcass and milk fat (Taniguchi et al., 2004; Mele et al., 2007; Moioli et al., 2007). Carta et al. (2006) performed a QTL analysis for the FA profile of sheep milk in an experimental Sarda $\times$ Lacaune back-cross population and identified a QTL for the ratio of CLA/VA in ovine chromosome 22 (OAR22), which coincided with the position of the $S C D$ gene. These authors suggested that this effect could be explained by the $S C D$ gene.

The genetic component of milk FA content was also investigated by Carta et al. (2008). Based on the estimates of repeatabilities, those authors suggested that a certain amount of additive genetic variance is 
available for selection purposes for most FA in sheep. However, in Churra sheep these traits have been shown to have low heritabilities (Sánchez et al., 2010). In the current study, we analyzed a commercial population of Spanish Churra sheep to detect QTL on OAR22 that were influencing the milk FA profile traits. Churra is an indigenous, highly specialized milk production breed farmed in the northwestern Spanish region of Castilla y León, where the traditional breeding system is based on indigenous grazing breeds. A selection scheme for milk production traits for this breed was begun in 1985 (De la Fuente et al., 1995). Currently, the scheme has a selection nucleus of about 40,000 animals belonging to 60 flocks, with production data available from milkrecording databases. For this study, initially, we carried out a QTL analysis on OAR22 for a total of 22 traits related to milk FA composition and 3 other production traits, following a daughter design. Furthermore, we assessed the possible role of the $S C D$ gene in the phenotypic variation of the milk FA profile observed in Churra sheep. For this purpose, we carried out an association analysis of 4 allelic variants previously identified in the ovine $S C D$ gene (García-Fernández et al., 2009) and the traits under study.

\section{MATERIALS AND METHODS}

\section{Resource Population}

The population analyzed in the present study included 799 ewes of the Spanish Churra breed distributed in 15 half-sib families and belonging to 16 different flocks. All animals belonged to the selection nucleus of the National Association of Churra Breeders (ANCHE) and were bred by AI. The average family size was around 53, ranging from 29 (family 3 ) to 131 (family 5) daughters per sire.

\section{Phenotypic Data}

The phenotypic traits considered in the study included 3 milk production traits (milk protein percentage, milk fat percentage, and milk yield) and 22 measurements related to the milk FA composition. These variables have been described previously by De la Fuente et al. (2009) and included 15 individual FA contents (quantified as grams per $100 \mathrm{~g}$ of total FA); 3 groups of FA based on the saturation level (SFA, MUFA, and PUFA); and 4 FA indexes (n-6/n-3, C14:1/C14:0, C16:1/C16:0, and CLA/VA). The full list of analyzed traits is provided in Table 1.

Data for the production traits were obtained from the records collected routinely by ANCHE in the official milk recording process and were measured as test-day records as explained by Gutiérrez-Gil et al. (2009).

Milk FA composition was determined as described by De la Fuente et al. (2009). Briefly, after extraction of total fat milk, which was carried out using the method described by Bligh and Dyer (1959), the FA content was determined by gaseous chromatography after methylation of free FA with $\mathrm{NaOCH}_{3}$. For this measurement, a Hewlett Packard 6890 Series GC System chromatograph (Wilmington, DE) was used.

\section{Genotyping and Linkage Map}

DNA was extracted from whole blood of ewes and semen of sires following standard methods detailed in García-Fernández et al. (2009). Eight microsatellite markers that were evenly distributed across OAR22 were selected from the published ovine linkage map (Maddox et al., 2001). These microsatellites were BMS651, FAS, HEL11, D9M1722, INRA81, TGLA429, MAF36, and $M C M A 73$. Fluorescence-labeled primers were used to amplify these markers in 3 separate groups. Two multiplex PCR reactions were performed in a final volume of $10 \mu \mathrm{L}$, containing $25 \mathrm{ng}$ of DNA and $6.5 \mu \mathrm{L}$ of Taq PCR Master Mix (Qiagen, Valencia, CA). Marker TGLA429 was amplified individually following a standard PCR protocol. After a $95^{\circ} \mathrm{C}$ incubation step for $15 \mathrm{~min}$, amplifications were performed for a total of 30 cycles using the following conditions: denaturation at $94^{\circ} \mathrm{C}$ for $30 \mathrm{~s}$; annealing at $50^{\circ} \mathrm{C}, 55^{\circ} \mathrm{C}$, or $60^{\circ} \mathrm{C}$ for $90 \mathrm{~s}$; and extension at $72^{\circ} \mathrm{C}$ for $60 \mathrm{~s}$, with a final extension step of $60 \mathrm{~min}$ at $72^{\circ} \mathrm{C}$. Different volumes of each PCR reaction were mixed, and samples were then processed on an ABI3130 DNA Analyzer (Applied Biosystems, Foster City, CA). Allele identification was performed with the software GeneMapper 4.0 (Applied Biosystems).

In addition, 4 SNP markers identified in the ovine $S C D$ gene were genotyped in all the animals included in the study. These SNP were located in the $5^{\prime}$ untranslated region of the $S C D$ gene (SNP_SCD01), intron 2 (SNP_SCD02 and SNP_SCD03), and intron 3 (SNP_SCD04). A detailed description of the genotyping methods for these SNP has been provided by García-Fernández et al. (2009). Briefly, SNP_SCD02, SNP_SCD03, and SNP_SCD04 were genotyped using TaqMan technology (Qiagen), and SNP_SCD01 was genotyped by direct sequencing.

Initially, a linkage map including the microsatellite markers analyzed on OAR22 was constructed using Crimap v2.4 software (Green et al., 1990). A second linkage map was built by including the 4 SNP analyzed in the $S C D$ gene. 
Table 1. List of traits analyzed in the present work and the significant associations detected in the association analysis performed with 4 stearoyl CoA-desaturase $(S C D)$ allelic variants described by García-Fernández et al. (2009)

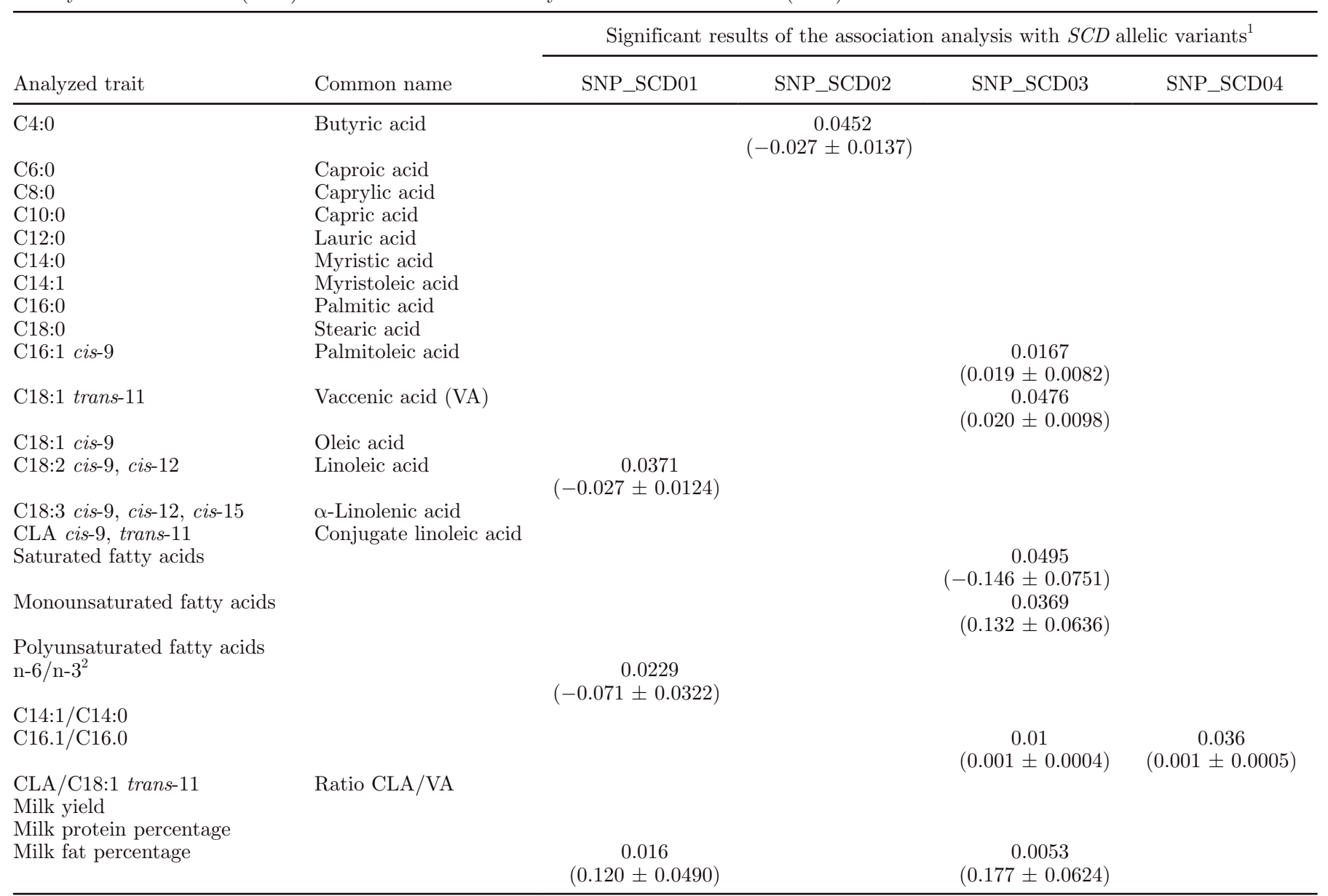

\footnotetext{
${ }^{1}$ The allelic substitution effects and their standard error are indicated in parentheses.
}

${ }^{2} \mathrm{n}-6: \mathrm{n}-3$ ratio $=($ linoleic acid $+\gamma$-linoleic acid + arachidonic acid $) / \alpha$-linoleic acid.

\section{QTL Analysis}

The dependent variables for the QTL analysis were yield deviations (YD) corresponding to each of the traits under study. These quantities were estimated as averages of the ewes' raw phenotypic records corrected for fixed environmental effects that had previously been found to have a significant effect on the traits under study (De la Fuente et al., 2009). In addition, records were corrected for permanent ewe environmental effect, as indicated by VanRaden and Wiggans (1991). The computation of YD was conducted using univariate animal repeatability models. The particular fixed effects considered were herd-test-day effect (HTD, 157 levels), the effect of days in milk (DIM, 7 levels) and the age of the ewe at parturition (AGE, 6 levels). Yield deviations of animal $l$ were computed using the following equation:

$$
Y D_{l}=\frac{\sum_{1}^{n_{l}} y_{i j k l}-\widehat{H T D}_{i}-\widehat{D I M}_{j}-\widehat{A G E}_{k}-\hat{p}_{l}}{n_{l}},
$$

where $n_{l}$ is the number of test-day records measured on each animal $l$, and $\hat{p}_{l}$ is the prediction of the permanent environmental effect for animal $l$. Yield deviations were calculated based on a total of 4,100 records from the 976 Churra ewes for which milk FA composition records were available. Although only 799 of these ewes had been genotyped for the markers studied in OAR22, the complete database of animals with FA measurement has been used in the estimation of YD.

The QTL mapping was performed using the webbased software package Grid-QTL (Seaton et al., 2006), which implements the multi-marker linear regression 
method for half-sib designs described by Knott et al. (1996). The information content (IC) across the map length was also calculated with this program.

An initial QTL analysis was performed using the linkage map including only the microsatellite markers. For each of the traits under study, a least squares analysis was performed at $1 \mathrm{cM}$ across the linkage map. Chromosome-wise significance thresholds for each of the traits under analysis were determined by a 10,000-permutation test (Churchill and Doerge, 1994). The bootstrapping method was used to calculate the 95\% confidence interval (CI) for the QTL locations (Visscher et al., 1996).

For each QTL identified at the whole population level, a within-family QTL analysis was performed to identify the segregating families (chromosome-wise $P$ value, $P_{\mathrm{c}}<0.10$ obtained by permutation testing). Estimations of the QTL allelic substitution effect for the segregating families were obtained from the withinfamily QTL analyses. The proportion of the phenotypic variance explained by the QTL $\left(\sigma_{Q T L}^{2} / \sigma_{P}^{2}\right)$ was calculated as $4 \times[1(1-$ mean square full model $) /($ mean square reduced model)] (Knott et al., 1996), where $\sigma_{Q T L}^{2}$ is the additive variance at the QTL and $\sigma_{P}^{2}$ is the phenotypic variance. To clarify the role of 4 SNP detected in the $S C D$ gene (García-Fernández et al., 2009), we performed additional analyses in which we examined whether these polymorphisms, to some extent, could explain the variation assigned to the chromosomal regions identified. In this analysis, the phenotypes were corrected for systematic environmental effects and additionally corrected for the known $S C D$ genotypes for each SNP by including these genotypes as a fixed effect in the model.

For calculation of the statistical power of the experiment, we followed the method of Weller et al. (1990). Hence, the statistical power of our experiment to detect a QTL with a substitution effect of 0.25 phenotypic SD units and 2 alleles at equal frequency and affecting a trait with a heritability of 0.05 (considering the last estimates for FA composition traits in Churra sheep (Sánchez et al., 2010) was estimated to be $20 \%$. This was assuming a type I error rate of 0.05 , a $9 \%$ recombination frequency between the QTL and marker, and that half of the analyzed sires were heterozygous at the QTL.

\section{Association Analysis}

The effect of the 4 genotyped $S C D$ gene SNP on milk FA composition and milk production traits was estimated individually assuming the following univariate sire repeatability model:

$$
\begin{aligned}
y_{i j k l m n} & =H T D_{i}+D I M_{j}+A G E_{k}+s_{m} \\
& +p_{l}+\beta \times S N P+e_{i j k l m n},
\end{aligned}
$$

where the new terms are $s_{m}$ referring to the ram $m$ siring ewe $l, \beta$ referring to the allele substitution effect for the particular SNP studied at this time, and SNP referring to a covariate reflecting the number of copies of a particular allele that the animal $l$ is carrying at the studied locus (e.g., 1, GG; 2, AG; or 3, AA).

The estimation of variance components was done with the program REMLF90 (Misztal, 1998). Once the variance components were estimated by a REML procedure, a fixed model equivalent to the described previously mixed model was implemented to test for the significance of the allele substitution effect. This was conducted using an $F$-test, which was repeated 4 times (once for each of the studied SNP) for every trait under study using R (R Development Core Team, 2008). In the fixed equivalent model, the covariance structure between records produced by the same female and between those measurements from half-sibs was absorbed in the residual (co)variance matrix. In contrast to the data set for the linkage analysis, only those records from females genotyped for at least 1 of the studied SNP were considered to calculate these parameters.

\section{RESULTS}

\section{Linkage Map}

The linkage map constructed for the Churra population studied in this experiment included 8 microsatellite markers and was in agreement with the published map (Maddox et al., 2001). A graphical representation of marker distribution, as well as the IC along the $87 \mathrm{cM}$ (Kosambi) length of the obtained linkage map is shown in Figure 1. The average IC along the map was $79 \%$, ranging from $63.7 \%$ at $6 \mathrm{cM}$ (between the BMS651 and FAS markers) to $93.7 \%$ at $42 \mathrm{cM}$ (between the markers D9M1722 and INRA81).

The map including the SNP of the $S C D$ gene was $87.6 \mathrm{cM}$ long, with the position of the $S C D$ SNP that were analyzed coinciding with microsatellite marker D9M1722 (41.6 cM). According to the Virtual Sheep Genome Assembly v2.0 (Dalrymple et al., 2007; http:// www.livestockgenomics.csiro.au/perl/gbrowse.cgi/ vsheep2/), as consulted in July 2009, this microsatellite maps approximately $68.3 \mathrm{kbp}$ downstream of the $S C D$ gene.

Regarding the variability displayed for the $4 S C D$ $\mathrm{SNP}$ in the population, minor allelic frequencies were 0.372, 0.191, 0.193, and 0.189 for SNP_SCD01 (C/A), SNP_SCD02 (A/G), SNP_SCD03 (T/C), and SNP_ 


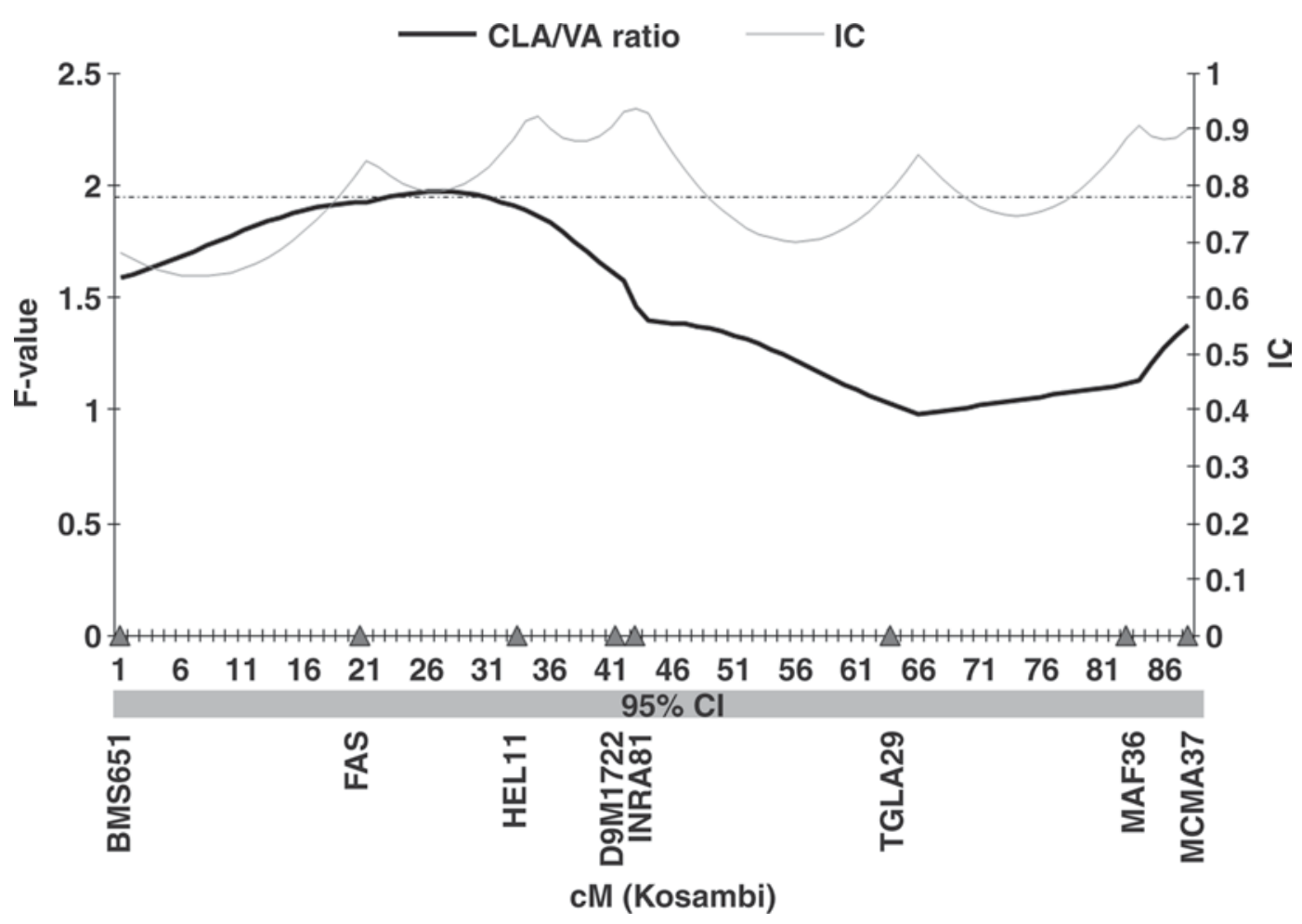

Figure 1. Across-family statistical profile obtained in ovine chromosome 22 for the conjugated linoleic acid/vaccenic acid ratio (CLA/VA) in the Churra sheep population analyzed in the present study. The $F$-statistic obtained in the regression analysis is represented across the linkage map of the studied chromosome on the left y-axis. The horizontal dashed line represents the $5 \%$ chromosome-wise significance threshold. Information content (IC) obtained along the linkage groups is shown on the right y-axis. Beginning at the centromeric end, the triangles on the $\mathrm{x}$-axis indicate the relative positions of the markers analyzed in this chromosome. The confidence interval (95\% CI) calculated by bootstrapping analysis for the CLA/VA ratio QTL is shown as a dark gray box at the bottom of the figure.

SCD04 (G/A), respectively. Concerning the phases displayed by the 15 rams, a total of 6 different haplotypes were detected distributed as follows: rams 1,3 , and 4 : CATG/CATG; rams 7 and 14: AACG/AACG; ram 2: CGTA/CGTA; rams 5 and 10: AACG/CATG; ram 11: AACG/CGTA; ram 6: AATG/CGTG; ram 9: CGTA/ AATG; rams 8 and 12: CGTA/CATG, and rams 13 and 15: AATG/CATG.

\section{QTL Detection}

The across-family regression analysis performed for the 25 traits analyzed on OAR22 identified only a borderline significant QTL at the $5 \%$ chromosome-wise level for the CLA/VA ratio at position $26 \mathrm{cM}(P=$ 0.049; Table 2; Figure 1). The average percentage of the phenotypic variance explained by the identified QTL in the across-family analysis was $10.91 \%$. The $95 \%$ CI calculated for this QTL effect involved the entire chromosomal length (0.0 to $87.0 \mathrm{cM}$ ). None of the other analyzed traits showed a significant effect, although there was certain evidence of linkage association at the $10 \%$ chromosome-wise significance level $(P=0.089)$ for
CLA content at position $27 \mathrm{cM}$ and for PUFA content at position $2 \mathrm{cM}(P=0.0673)$. All of these results regarding significance must be interpreted with caution, however, because no adjustments were made to account for applying the analysis to a large number of correlated traits.

Based on the putative QTL identified for the CLA/ VA ratio at the whole-population level, we performed an individual regression analysis of this trait for the different families. The results of this within-family analysis, which are summarized in Table 2 and depicted in Figure 2 , revealed two $5 \%$ chromosome-wise significant QTL segregating in families 3 and 6 , at positions 33 and 42 cM, respectively. The $S C D$ gene maps within the flanking interval of the QTL identified in family 6, HEL11DM91722, which is $7.6 \mathrm{cM}$ long. The QTL identified in family 3 at position $33 \mathrm{cM}$ was the most significant association revealed by the within-family analysis and was located within the marker interval FAS-HEL11, $8.2 \mathrm{cM}$ away from the $S C D$ gene position. For one other family, family 4 , the maximum $F$-test for the CLA/VA statistical profile was localized at position $20 \mathrm{cM}$, at the $10 \%$ chromosome-wise significance level. However, the 
Table 2. Linkage association identified at the $5 \%$ chromosome-wise significance level in the regression analysis of ovine chromosome 22 in the commercial population of Churra sheep analyzed in the present study

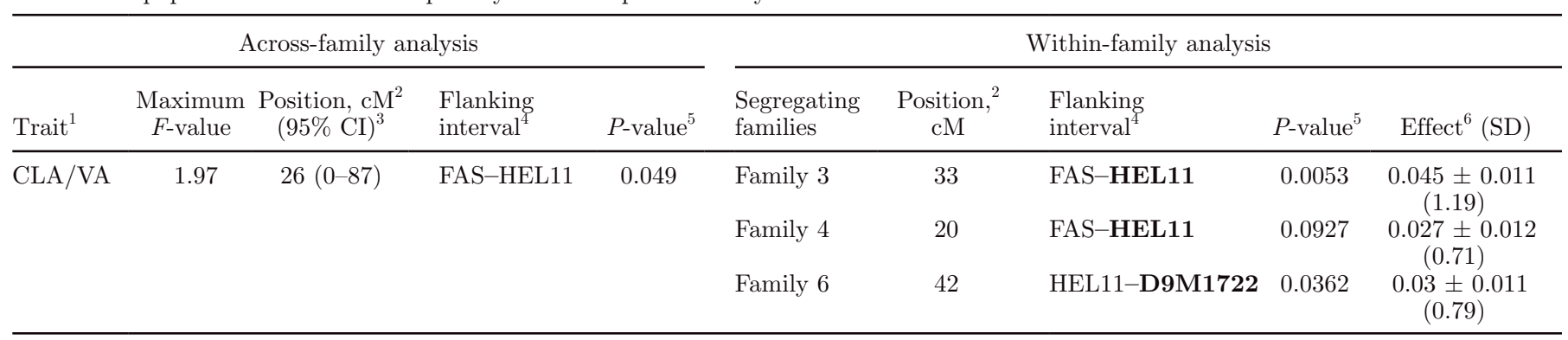

${ }^{1}$ Trait: CLA/VA = ratio between conjugated linoleic acid and vaccenic acid contents.

${ }^{2}$ Position (cM Kosambi) of the chromosome where the maximum $F$-statistic value was obtained in the across- and within-family analyses.

${ }^{3}$ The $95 \%$ confidence interval obtained by bootstrapping analysis (Visscher et al., 1996) is shown in parentheses (cM Haldane).

${ }^{4}$ Markers flanking the position of the maximum $F$-statistic; markers in bold are $<1 \mathrm{cM}$ from the maximum $F$-statistic.

${ }^{5}$ Chromosome-wise $P$-value associated with the maximum $F$-statistic value of the across-family and within-family analyses, calculated through a chromosome-wise permutation test (Churchill and Doerge, 1994) implemented through Grid QTL (http://www.gridqtl.org.uk/).

${ }^{6}$ Magnitude of the allelic substitution effect calculated for each segregating family, expressed in units of the trait (g of CLA/g of VA) and in phenotypic SD units (in parentheses).

$F$-ratio showed a kind of plateau from positions 0 to 24 cM (Figure 1), which seems to be the result of the lack of informativeness of the first marker analyzed in this chromosome for family 4 . The estimates of the QTL in the 3 segregating families ranged from 0.71 (family 4 ) to 1.19 (family 3) standard deviations of the YD.
When the analysis was repeated with the SNP of the $S C D$ gene in the map, the result of the across-family analysis was very similar to the analysis with only microsatellites in the map, with the CLA/VA QTL peak localized at position $26 \mathrm{cM}$ and reaching a similar statistical significance $(P=0.0484)$. With the inclusion of

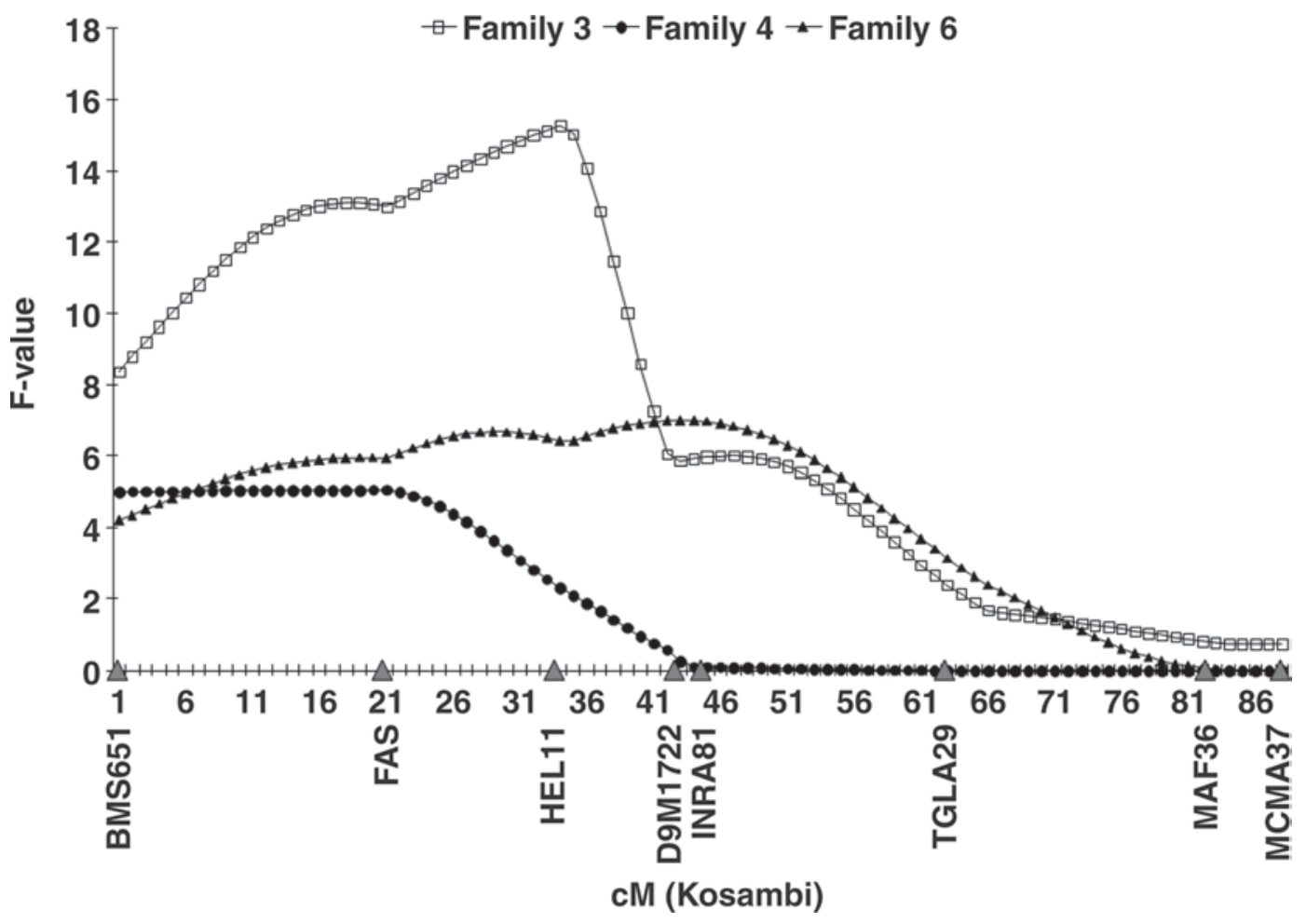

Figure 2. Test statistics curves obtained from the within-family analysis of the conjugated linoleic acid/vaccenic acid ratio (CLA/VA) for the 3 segregating families. Beginning at the centromeric end, the triangles on the $\mathrm{x}$-axis indicate the relative positions of the markers analyzed in this chromosome. 
these SNP as fixed effects in the model performed on the CLA/VA ratio, there was no substantial reduction of the $F$-statistics, and the maximum $F$-value remained at the same position.

The within-family analysis of the CLA content revealed 3 segregating families for the linkage association identified for this trait in the across family-analysis, 2 of which were also segregating for the CLA/VA ratio QTL. For this suggested association, the significance levels and the position of the statistical profile peaks differed substantially among the 3 half-sib groups that seemed responsible of the effect detected in the acrossfamily analysis (family $3: P=0.0068,9 \mathrm{cM}$; family 4 : $P=0.0872,20 \mathrm{cM}$; family $10: P=0.097,83 \mathrm{cM})$. For the linkage association suggested at the proximal end of the chromosome for the PUFA content, none of the analyzed families showed chromosome-wise significant $P$-values $(P \approx 0.12$ to 0.15$)$. Therefore, the effect detected for this trait at the across-family level seems to be the result of low significant effects detected in several families.

\section{Association Analysis}

The association analyses performed with the $4 S C D$ polymorphisms reported by García-Fernández et al. (2009) revealed 11 significant associations at the 5\% nominal level, which are summarized in Table 1. The polymorphism located in the promoter region of the $S C D$ gene, SNP_SCD01, showed a significant effect on fat percentage, the proportion of linoleic acid (C18:2 cis-9,cis-12), and the $\mathrm{n}-6 / \mathrm{n}-3$ ratio. The allelic variant SNP_SCD02, which is localized in intron 2 of the $S C D$ gene, showed a significant effect on butyric acid (C4:0). The third allelic variant genotyped, SNP_SCD03, is located in intron 2 and significantly influenced 6 of the analyzed traits: palmitoleic acid (C16:1), vaccenic acid (C18:1 trans-11), SFA and MUFA contents, C16:1/ C16:0 ratio, and milk fat percentage. The SNP located in intron 3, SNP_SCD04, only showed significant effects on C16:1/C16:0 ratio. When a Bonferroni correction was performed to take into account the multiple traits and SNP analyzed, none of the associations remained significant $(P<0.0041)$. It is important to note that for this correction, a total of 4 independent traits and 3 independent SNP were considered because in a principal components analysis performed using $\mathrm{R}$ software (R Development Core Team, 2008), 4 trait factors accounted for $91.54 \%$ of the total variance of the phenotypes under study (data not shown), whereas 3 SNP were estimated as the effective number of markers required to explain the $97.5 \%$ of the genotype variation based on the method proposed by Gao et al. (2009).

\section{DISCUSSION}

Based on the known biological function of SCD and the previously reported associations of the $S C D$ gene with FA composition of ruminant products, including sheep milk, a commercial population of Spanish Churra sheep was analyzed in this study to detect regions of ovine chromosome 22 harboring genes underlying milk FA and milk production-related traits.

We identified a suggestive 5\% chromosome-wise significant QTL $(P<0.05)$ influencing the CLA/VA ratio with its maximum peak located at $26 \mathrm{cM}$ of the studied chromosome. The within-family analysis detected 2 families clearly segregating for this QTL (families 3 and 6). The position of the QTL detected in Churra sheep for this genetic effect $(26 \mathrm{cM})$ differs from the QTL identified in the Sarda $\times$ Lacaune experimental population analyzed by Carta et al. (2006), which coincided with the position of the $S C D$ gene $(35 \mathrm{cM}$ in the Sarda $\times$ Lacaune map and $40 \mathrm{cM}$ in the Churra map). We should take into account, however, the limited accuracy of low-density linkage studies to estimate the position of QTL effects under half-sib designs (Gutiérrez-Gil et al., 2007). This is especially true in commercial populations where multiple QTL alleles affecting the same trait may be segregating in the different analyzed families and where the marker informativeness may differ between families. Hence, it is possible that the poor resolution of the regression analysis along the first marker interval for the less significant family (family 4) may have biased the position of the QTL in the across-family analysis. Hence, the discrepancies in the within-family QTL peak positions may have negatively affected the accuracy of the QTL peak position at the across-family level. In addition, the QTL identified for the CLA/VA ratio in Churra sheep seems to be segregating at a low frequency (3 of 15 sires were segregating), which is an important limiting factor affecting the statistical power of our analysis.

On the other hand, when assessing the alleged role of the $S C D$ gene in the CLA/VA ratio QTL detected in Churra sheep, we must consider the following facts. First, the low variability identified in this gene for this sheep breed is surprising. In a previous work in which we sequenced a total of $4,338 \mathrm{bp}$ of the $S C D$ gene for the 15 Churra sires used in this analysis and 24 additional unrelated Churra ewes (García-Fernández et al., 2009), no allelic variants were identified in the protein coding segments of the sequence; only 4 polymorphisms were identified in the noncoding region of the gene. From a functional point of view, using the web-based Cis-element Cluster Finder software (http://zlab. bu.edu/ mfrith/cister.shtml), we have not found any 
regulatory cis elements that could mediate the expression of the $S C D$ gene in the sequences harboring any of the 4 studied SNP. Nevertheless, it is possible that some mutations affecting the control of $S C D$ gene expression in the mammary gland were localized in the unexplored region of this gene. Second, the correction for $S C D$ genotypes did not eliminate the previously significant QTL affecting the CLA/VA ratio, which suggests that these mutations are neither directly responsible of the detected effect nor in linkage disequilibrium with the causal mutation. This observation is consistent with the fact that the association analysis of the 4 mentioned SNP genotypes with the CLA/VA ratio did not show any significant association. In addition, no significant results were identified in our study for other FA ratios directly related to SCD activity (C14:1/C14:0; C16:1/ C16:0). Based on these observations, the $S C D$ gene does not appear to be directly responsible for the reported suggestive QTL.

Considering the low significance level of the linkage association detected, the first step of further studies should be focused on confirmation of the borderline significant effect reported herein. An increase of the statistical power of the experiment, especially by increasing the size of the analyzed families, is highly desirable to reach a reliable conclusion about the existence of a genuine QTL influencing milk FA composition on chromosome 22 in Churra sheep. If the QTL were confirmed, a fine-mapping approach would be required to redefine the QTL position and to get a reduction of the 95\% CI, which would facilitate the identification of appropriate candidate genes. In this regard, it should be noted that, based on the Sheep Genome Assembly 1.0 (http://www.livestockgenomics.csiro.au/perl/gbrowse. cgi/oar1.0/), the region where the peak of the putative QTL reported here was found, close to the FAS microsatellite, harbors some genes related to lipid metabolism such as lysosomal acid lipase ( $L I P A)$ and gastric lipase $(L I P F)$. Lysosomal acid lipase is a critical enzyme in the hydrolysis of cholesteryl esters and triglycerides to generate free FA and cholesterol in the lysosome, whereas gastric lipase has an important role in the digestion of triglycerides in the gastrointestinal tract, especially in patients suffering from pancreatic lipase deficiencies (Carriere et al., 2005). To our knowledge, none of these genes has been previously associated with the CLA synthesis pathway.

Regarding the association analysis performed for the $4 S C D$ SNP genotyped in the Churra population, a few significant associations were identified at the nominal level for the considered $S C D$ polymorphisms. Although none of the associations identified in this analysis at the nominal significance level remained significant when a stringent Bonferroni correction was applied, this cor- rection dramatically increases the risk of committing type II errors (false negatives; Nakagawa, 2004). Taking into account the loss of power because of this very conservative correction, we consider it important to report the regions detected at the nominal significance level in initial analyses. Nevertheless, an independent confirmation of these primary results will be required before attempting its practical implementation in selection programs.

The most remarkable association identified by our association analysis was that detected between SNP_SCD03 and milk fat percentage, as it was close to the significance threshold established by the stringent Bonferroni correction $(P=0.0053)$. To confirm the real nature of this association in Churra sheep, an additional analysis based on the paternal and maternal inherited haplotypes reconstructed using the phase software (Stephens et al., 2001) was performed. The $S C D$-haplotype effects were estimated by replacing in the model described for the association study the SNP effect by a vector indicating which haplotypes and how many copies were carried by the ewe. This analysis showed some borderline effects of $S C D$ haplotypes on linoleic acid content and the $\mathrm{C} 16: 1 / \mathrm{C} 16: 0$ ratio, as well as a clear influence on milk fat percentage. Based on the results reported herein, the effect of the $S C D$ gene on milk fat percentage in Churra sheep seems to be genuine. This is an interesting association when the influence of this trait on cheese yield is considered (Rudan et al., 1999). As for the other results identified in the association analysis, this effect was not picked up by the linkage analysis. In this regard, we should take into account the limitations of our linkage analysis, where the analyzed sires were assumed to be heterozygous $(Q q)$ at the QTL position. These analyses only model a paternal QTL component and ignore information from the maternal alleles or any genetic links between half-sib families (de Koning, 2006). Thus, using association analysis to assess the direct effect of the genotype of 1 SNP on a trait may identify associations that do not satisfy this assumption (Borecki and Suarez, 2001). In cattle, certain evidence of association between an amino acid substitution in the bovine $S C D 1$ gene (Ala293Val) and milk fat content was reported in Canadian Jersey cows, whereas the same polymorphism showed a clear effect on 305-d milk and protein yields (Kgwatalala et al., 2009). Further studies are needed to better understand the direct influence of the $S C D$ gene on milk production traits.

\section{CONCLUSIONS}

Our across-family linkage analysis identified a suggestive QTL at the chromosome-wise level in the first 
half of chromosome 22 influencing the CLA/VA ratio. We investigated the extent to which the detected QTL could be explained by polymorphisms in the $S C D$ gene. The correction for $4 S C D$ SNP did not decrease the proportion of phenotypic variance explained by the QTL. Based on this, the $S C D$ gene seems not to have a direct influence on the genetic effect reported in the commercial population of Churra sheep studied here. A confirmation of this suggestive significant genetic effect is required before addressing further research efforts on this region. The association analysis performed with the $4 S C D$ SNP considered in this study suggests a possible effect of the $S C D$ gene on milk fat percentage in Churra sheep, which should be confirmed in independent analyses.

\section{ACKNOWLEDGMENTS}

This work was supported by the Spanish Ministry of Science (Project AGL2005-04321) and the Castilla and León regional government (Junta de Castilla y León) by a grant for research groups of excellence (Project GR43). Marta García-Fernández is funded by a FPI contract from the Spanish Ministry of Science. Beatriz Gutiérrez-Gil is funded by the "Juan de la Cierva Program" of the Spanish Ministry of Science. Elsa GarcíaGámez is funded by a contract from Junta de Castilla y León.

\section{REFERENCES}

Bauman, D. E., L. H. Baumgard, B. A. Corl, and J. M. Griinari. 1999 Biosynthesis of conjugated linoleic acid in ruminants. In Proceedings of the American Society of Animal Science, Indianapolis, IN. http://www.asas.org/jas/symposia/proceedings/0937.pdf

Bauman, D. E., I. H. Mather, R. J. Wall, and A. L. Lock. 2006. Major advances associated with the biosynthesis of milk. J. Dairy Sci. 89:1235-1243.

Bligh, E. G., and W. J. Dyer. 1959. A rapid method of total lipid extraction and purification. Can. J. Biochem. Cell Biol. 37:911917.

Borecki, I. B., and B. K. Suarez. 2001. Linkage and association: Basic concepts. Adv. Genet. 42:45-66.

Cabiddu, A., M. Decandia, G. Molle, G. Pinna, M. Addis, S. Spada, A. Pirisi, and G. Piredda. 2003. Effects of different pasture on CLA content in sheep milk and cheese. Ital. J. Anim. Sci. 2(Suppl. 1):518-520.

Cabiddu, A., M. Decandia, M. Addis, G. Piredda, A. Pirisi, and G. Molle. 2005. Managing Mediterranean pastures in order to enhance the level of beneficial fatty acids in sheep milk. Small Rumin. Res. 59:169-180.

Carriere, F., P. Grandval, C. Renou, A. Palomba, F. Prieri, J. Giallo, F. Henniges, S. Sander-Struckmeier, and R. Laugier. 2005. Quantitative study of digestive enzyme secretion and gastrointestinal lipolysis in chronic pancreatitis. Clin. Gastroenterol. Hepatol. 3:28-38.

Carta, A., S. Casu, M. G. Usai, M. Addis, M. Fiori, A. Fraghì, S. Miari, L. Mura, G. Piredda, L. Schibler, T. Sechi, J. M. Elsen, and F. Barillet. 2008. Investigating the genetic component of fatty acid content in sheep milk. Small Rumin. Res. 79:22-28.
Carta, A., T. Sechi, M. G. Usai, M. Addis, M. Fiori, A. Fraghì, S. Miari, L. Mura, G. Piredda, J. M. Essen, L. Schibler, F. Barrillet, and S. Casu. 2006. Evidence for a QTL affecting the synthesis of linoleic conjugated acid cis-9, trans-11 from 11-c 18:1 acid on ovine chromosome 22. Proc. 8th World Congress on Genetics Applied to Livestock Production. Belo Horizonte, Brazil. Commun. no. 12-03. Instituto Prociencia, Belo Horizonte, Brazil.

Churchill, G. A., and R. W. Doerge. 1994. Empirical threshold values for quantitative trait mapping. Genetics 138:963-971.

Dalrymple, B. P., E. F. Kirkness, M. Nefedov, S. McWilliam, A. Ratnakumar, W. Barris, S. Zhao, J. Shetty, J. F. Maddox, M. O'Grady, F. Nicholas, A. M. Crawford, T. Smith, P. J. de Jong, J. McEwan, V. H. Oddy, and N. E. Cockett. 2007. Using comparative genomics to reorder the human genome sequence into a virtual sheep genome. Genome Biol. 8:R152.

de Koning, D. J. 2006. Conflicting candidates for cattle QTLs. Trends Genet. 22:301-305.

De la Fuente, L. F., E. Barbosa, J. A. Carriedo, C. Gonzalo, R. Arenas, and J. M. Fresno. 2009. Factors influencing variation of fatty acid content in ovine milk. J. Dairy Sci. 92:3791-3799.

De la Fuente, L. F., G. Fernández, and F. San Primitivo. 1995. Breeding programme for the Spanish Churra sheep breed. Cahiers Opt. Medit. 11:165-172.

Gao, X., L. C. Becker, D. M. Becker, J. Starmer, and M. A. Province. 2009. Avoiding the high Bonferroni penalty in genome-wide association studies. Genet. Epidemiol. doi:10.1002/gepi.20430.

García-Fernández, M., B. Gutiérrez-Gil, E. García-Gámez, and J. J. Arranz. 2009. Genetic variability of the stearoyl-CoA desaturase gene in sheep. Mol. Cell. Probes 23:107-111.

Green, P., K. Falls, and S. Crooks. 1990. Documentation for CRI-MAP version 2.4. Washington University School of Medicine, St. Louis, MO.

Gutiérrez-Gil, B., M. F. El-Zarei, L. Alvarez, Y. Bayón, L. F. de la Fuente, F. San Primitivo, and J. J. Arranz. 2009. Quantitative trait loci underlying milk production traits in sheep. Anim. Genet. 40:423-434.

Gutiérrez-Gil, B., M. F. El-Zarei, Y. Bayón, L. Álvarez, L. F. de la Fuente, F. San Primitivo, and J. J. Arranz. 2007. Short communication: Detection of quantitative trait loci influencing somatic cell score in Spanish Churra sheep. J. Dairy Sci. 90:422426.

Jensen, R. G., A. M. Ferris, and C. J. Lammi-Keefe. 1990. The composition of milk fat. J. Dairy Sci. 74:3228-3243.

Kgwatalala, P. M., E. M. Ibeagha-Awemu, A. F. Mustafa, and X. Zhao. 2009. Influence of stearoyl-coenzyme A desaturase 1 genotype and stage of lactation on fatty acid composition of Canadian Jersey cows. J. Dairy Sci. 92:1220-1228.

Knott, S. A., J. M. Elsen, and C. S. Haley. 1996. Methods for multiplemarker mapping of quantitative trait loci in half-sib populations. Theor. Appl. Genet. 93:71-80.

Maddox, J. F., K. P. Davies, A. M. Crawford, D. J. Hulme, D. Vaiman, E. P. Cribiu, B. A. Freking, K. J. Beh, N. E. Cockett, N. Kang, C. D. Riffkin, R. Drinkwater, S. S. Moore, K. G. Dodds, J. M. Lumsden, T. C. van Stijn, S. H. Phua, D. L. Adelson, H. R. Burkin, J. E. Broom, J. Buitkamp, L. Cambridge, W. T. Cushwa, E. Gerard, S. M. Galloway, B. Harrison, R. J. Hawken, S. Hiendleder, H. M. Henry, J. F. Medrano, K. A. Paterson, L. Schibler, R. T. Stone, and B. van Hest. 2001. An enhanced linkage map of the sheep genome comprising more than 1,000 loci. Genome Res. 11:1275-1289.

Mele, M., G. Conte, B. Castiglioni, S. Chessa, N. P. P. Macciotta, A. Serra, A. Buccioni, G. Pagnacco, and P. Secchiari. 2007. Stearoyl-CoA desaturase gene polymorphism and milk fatty acid composition in Italian Holsteins. J. Dairy Sci. 90:4458-4465.

Misztal, I. 1998. REMLF90. Manual. Available at: http://nce.ads.uga. edu/ ignacy/numpub/blupf90/docs/remlf90.pdf. Accessed April $20,2009$.

Moioli, B., G. Contarini, A. Avalli, G. Cavillo, L. Orr, G. De Matteis, G. Masoero, and F. Napoletano. 2007. Effect of stearoyl coenzyme 
A desaturase polymorphism on fatty acid composition of milk. J. Dairy Sci. 90:3553-3558.

Nakagawa, S. 2004. A farewell to Bonferroni: The problems of low statistical power and publication bias. Behav. Ecol. 15:10441045.

Ntambi, J. M. 1995. The regulation of stearoyl-CoA desaturase (SCD). Prog. Lipid Res. 34:139-150.

Pariza, M. W., Y. Park, and M. E. Cook. 2000. Mechanisms of action of conjugated linoleic acid: Evidence and speculation. Proc. Soc. Exp. Biol. Med. 223:8-13.

R Development Core Team. 2008. R: A language and environment for statistical computing. R Foundation for Statistical Computing, Vienna. Austria. http://www.R-project.org. Accessed April 20, 2009.

Rudan, M. A., D. M. Barbano, J. J. Yun, and P. S. Kindstedt. 1999. Effect of fat reduction on chemical composition, proteolysis, functionality, and yield of Mozzarella cheese. J. Dairy Sci. 82:661-672.

Sánchez, J. P., F. San Primitivo, E. Barbosa, L. Varona, and L. F. de la Fuente. 2010. Genetic determination of fatty acid composition in Spanish Churra sheep milk. J. Dairy Sci. 93:330-339.

Seaton, G., J. Hernandez, J. A. Grunchec, I. White, J. Allen, D. J. De Koning, W. Wei, D. Berry, C. Haley, and S. Knott. 2006. GridQTL:
A Grid Portal for QTL Mapping of Compute Intensive Datasets. Proceedings of the 8th World Congress on Genetics Applied to Livestock Production. Belo Horizonte, Brazil. Instituto Prociencia, Belo Horizonte, Brazil.

Stephens, M., N. Smith, and P. Donnelly. 2001. A new statistical method for haplotype reconstruction from population data. Am. J. Hum. Genet. 68:978-989.

Taniguchi, M., T. Utsugi, K. Oyama, H. Mannen, M. Kobayashi, Y. Tanabe, A. Ogino, and S. Tsuji. 2004. Genotype of stearoyl-CoA desaturase is associated with fatty acids composition in Japanese Black cattle. Mamm. Genome 14:142-148.

VanRaden, P. M., and G. R. Wiggans. 1991. Derivation, calculation and use of international animal model information. J. Dairy Sci. 74:2737-2746.

Visscher, P. M., R. Thompson, and C. S. Haley. 1996. Confidence intervals in QTL mapping by bootstrapping. Genetics 143:10131020.

Weller, J. I., Y. Kashi, and M. Soller. 1990. Power of daughter and granddaughter designs for determining linkage between marker loci and quantitative trait loci in dairy cattle. J. Dairy Sci $73: 2525-2537$. 